\title{
On the Absoluteness of Time
}

\author{
Carmine Cataldo ${ }^{1}$ \\ ${ }^{1}$ Independent Researcher, $\mathrm{PhD}$ in Mechanical Engineering, Battipaglia (Salerno), Italy \\ Correspondence: Carmine Cataldo, Battipaglia (Salerno), Italy. E-mail: catcataldo@hotmail.it
}

Received: May 15, 2017

Accepted: May 28, 2017

Online Published: May 31, 2017

doi:10.5539/apr.v9n3p46

URL: https://doi.org/10.5539/apr.v9n3p46

\begin{abstract}
This paper aims to qualitatively summarize the results up until now obtained in investigating the compatibility between the absoluteness of time and several well-known phenomena, such as the alleged increase of the mean lifetime of muons and the so-called relativistic corrections for GPS, whose explanation is commonly provided by resorting to Einstein's Relativity. To make the discussion more flowing, we have herein preferred to completely avoid the writing of equations. All the analytical solutions, as well as several explicative figures, can be found in the first six articles cited in the references, drafted by the same author of this manuscript.
\end{abstract}

Keywords: Absoluteness of Time, Extra Dimensions, Relativistic Motion, Muons Mean Lifetime, General Relativity, GPS Relativistic Corrections, Gravitational Redshift

\section{Introduction}

All the models we have elsewhere discussed (Cataldo, 2016a, 2016b, 2017a, 2017b, 2017c, 2017d) start from hypothesizing a closed Universe, homogeneous and isotropic, belonging to the so-called oscillatory class $\left(\mathrm{O}_{1}\right.$ type in the Harrison classification) (Harrison, 1967). More precisely, we postulate a Universe that approximately evolves following a simple-harmonic motion, whose pulsation is equal to the ratio between the speed of light and the value of the mean radius, taken as reference (Cataldo, 2017b, 2017c). In spite of this, we consider the variations of cosmological distances as being exclusively metric: in other terms, we postulate that the amount of space between whatever couple of points remains the same with the passing of time. In particular, once hypothesized a variability over time of the Planck constant (Cataldo, 2017a; Seshavatharam et al., 2013a, 2013b), the Cosmological Redshift may be banally explained by taking into account the conservation of energy. The existence of at least a further spatial dimension is postulated: more precisely, the Universe in its entirety is imagined as being flat and identifiable with a four-dimensional ball. Although the Universe is to be considered as being globally flat, the space we are allowed to perceive, when we are at rest, is curved, since it is identified with a hyper-sphere whose radius depends on our state of motion (Cataldo, 2016a, 2017c). All the points are replaced by straight line segments: more precisely, what we perceive as being a point may actually be a straight-line segment crossing the centre of the 4-ball with which we identify our Universe (Cataldo, 2016b). Time is considered as being absolute: however, it is fundamental to underline how this strong assumption does not imply that instruments and devices of whatever kind, finalized to measure time, are not influenced by motion and gravity (Cataldo, 2017d).

\section{Motion and the Absoluteness of Time}

The Lorentz transformations can be considered, without any doubt whatsoever, as the backbone of the theory of Special Relativity. Nonetheless, both the conventional derivation of the transformations and the meaning commonly assigned to them have been often savagely criticized, to the extent that, despite an alleged empirical evidence, the whole Special Relativity, in several occasions, has been brought into question. Firstly, it is worth underlining that, as Lorentz himself was forced to admit at a later time (Lorentz, 1909), the transformations had been already conceived, several years before the publication of the famous paper (Lorentz, 1904), by someone else (Voigt, 1887). Secondly, the work of Lorentz was anything but concretely linked to relativistic issues, at least in the Einsteinian sense of the term. Very simply, Lorentz's aim fundamentally lay in finding some transformations able to formally make the Maxwell equations (Maxwell, 1873) invariant. On this subject, moreover, it has been proved how the Lorentz transformations are not the only ones able to preserve the formal validity of the Maxwell equations (Di Mauro et al., 1997).

We have elsewhere (Cataldo, 2016a) shown how the Lorentz transformations can be alternatively deduced, albeit with a different meaning, once some noteworthy hypotheses concerning our Universe have been assumed, among 
which the existence of at least a further spatial dimension and the absoluteness of time stand out. Our alternative deduction, what is more, allows us to overcome a well-known misleading problem related to the so-called time transformations. It is commonly said that, when the speed assumed by the mobile frame of reference is far less than that of light, the Lorentz transformations tend to the Galilean ones. In other terms, according to the previous assertion, Galilean Relativity should be interpreted as a particular case of the Einsteinian one. This is an erroneous conviction (Ghosal et al., 1961). In fact, it is easy to verify how no limitation turns out to be formally imposed, as far as the numerators of the time transformations are concerned, on the spatial coordinates. Therefore, since the above-mentioned coordinates can evidently assume arbitrarily large values, an unconditional identification of the Lorentz transformations with the Galilean ones, when the speed tends to zero, should be considered as being de facto impossible (Di Mauro et al., 1995).

The procedure we exploit to alternatively deduce the Lorentz transformations is fundamentally based upon the conservation of energy. Firstly, taking advantage of the hypotheses highlighted in the introduction, we can easily obtain the so-called mass-energy equivalence. Bearing in mind that, according to our models, each point may actually be a straight-line segment crossing the center of the four-dimensional ball with which we identify our Universe, we can state that what we perceive as being a translatory motion is nothing but a rotation around the center of the above-mentioned ball. We have elsewhere (Cataldo, 2017c) proven that, by virtue of the conservation of energy, the radial extension of any segment depends on its state of motion: the more the speed increases, the more the radial extension decreases. When a point (actually a segment) is at rest, the corresponding radial extension, obviously, equates the radius (of curvature) of the Universe: in other terms, the point is placed on the external hyper-surface. In no case can the local speed exceed that of light. Nonetheless, we can also define a virtual speed (the speed measured by an observer at rest) whose value is provided by the product between the local (real) speed and the relativistic factor. Very evidently, the virtual speed can exceed that of light, and it tends to infinity when the radial extension tends to zero (when the local speed tends to that of light). When a point, initially at rest, starts moving (when a segment, initially at rest, starts rotating), the radial extension undergoes a reduction: consequently, once considered a second point, placed at a certain angular distance from the first (the angular distance is meant as the one measured by an ideal observer placed at center of the 4-ball), the corresponding arc distance depends on the state of motion. Ultimately, time does not undergo any dilation whatsoever due to the motion: on the contrary, the arc distance between two generic points (the only one we can actually measure) is not symmetric, and it depends on the value of the speed.

The alleged increase of the lifetime of muons, although coherent with Special Relativity, may be easily explained avoiding time dilations. Muons evidently succeed in covering a distance clearly not compatible with their mean lifetime: this is irrefutable. On the one hand, we may admit that time, for muons, starts slowing down due to the high value of their speed, but on the other hand, and for the same reason, we may also imagine that, for muons, both the radial extension and the distances undergo a reduction (the phenomenon, according to our theory, is no longer restricted to the direction of the motion). In the latter case, the speed perceived by an observer at rest is greater than what it really is, and time does not undergo any dilation whatsoever. Two different explanations, one of which based upon the absoluteness of time, both fully compatible with the Lorentz transformations, that consequently, though, acquire a completely different meaning in the two cases.

\section{Gravity and the Absoluteness of Time}

We have elsewhere (Cataldo, 2017d) proposed a simple qualitative model, finalized to discuss the compatibility between gravity and the absoluteness of time. At the beginning, taking into account a global symmetry, matter is imagined as being evenly spread on the hyper-sphere with which we identify the Universe we are allowed to perceive (actually, according to our hypotheses, matter fills homogeneously the corresponding 4-ball in its entirety). Let's consider a circumference, belonging to the surface of the ball, and the corresponding center: in a curved space, obviously, the predicted radius (the ratio between the perimeter of the circumference and $2 \pi$ ) does not coincide with the measured one (related, as far as the scenario initially hypothesized is concerned, to the component $g_{11}$ of the Robertson - Walker metric tensor). We postulate that, if the center acquires a greater mass, the circumference undergoes a contraction, but the value of the measured radius (the measured distance between the center and whatever point of the circumference) remains exactly the same, as well as the corresponding angular distance (as perceived by an ideal observer placed at the center of the 4-ball) (Cataldo, 2017d). If all the available mass is ideally concentrated in a single point, we may discuss the so-called vacuum field solution. Let's consider now a test particle orbiting around a point with a constant angular distance (the path is circular). We can state that the more the above-mentioned point acquires mass, the more the orbit followed by the particle turns out to be reduced. In spite of this, the measured distance, according to our model (Cataldo, 2017d), remains the same (the proper 
radius, consequently, is no longer related, as far as the so-called vacuum field solution is concerned, to the component $\mathrm{g}_{11}$ of the Schwarzschild metric tensor) (Schwarzschild, 1919).

As we know, there are two kinds of so-called relativistic corrections for GPS (Global Positioning System). Special Relativity predicts that time, on satellites, should slow down by virtue of their (relative) motion: this phenomenon, that we consider as being merely apparent, has been discussed in the previous paragraph. On the contrary, General Relativity requires that time, on satellites, should flow faster than it does on the surface of the Earth (in other terms, clocks closer to a massive object should tick more slowly, so to say, than those located at a greater distance). According to our model, the more a gravitational singularity acquires mass, the more the time needed to cover a whole orbit, at a fixed angular distance, turns out to be reduced, in spite of the fact that, by virtue of our hypotheses, the proper radius remains exactly the same: consequently, the more a particle approaches a gravitational source, the more time turns out to be apparently dilated. Ultimately, once again, we may state that time dilation is nothing but a merely apparent phenomenon, exclusively related, as far as gravity is concerned, to the contraction of the orbits.

Now, let's suppose that we are not disposed to accepting such a situation. More precisely, let's imagine that, instead of admitting that the orbit drawn by a test particle undergoes a contraction, we prefer to hypothesize that, due to a gravitational source placed at the origin, time starts slowing down. By virtue of this interpretation, taking into account the fact that, coherently with our model, a light impulse takes the same time, with or without gravitational singularity, to cover the distance (once fixed the angular one) between the origin and any other point, we are forced to modify the value of the proper radius. The reason is very simple. On the one hand, the speed of light cannot be influenced by the singularity; on the other hand, we forcefully postulate that, due to the gravitational source, time starts to slow down (we refuse to admit that the orbit undergoes a contraction). As a consequence, in order to keep the speed of light constant, we have to imagine that the proper radius undergoes a dilation. In the light of this interpretation, we have elsewhere (Cataldo, 2017d) deduced a Schwarzschild-like metric (more precisely a Droste/Brillouin/Hilbert-like metric) without using General Relativity.

If we postulate the absoluteness of time, the so-called Gravitational Redshift, obviously, can no longer be legitimized by means of time dilation. To explain the above-mentioned phenomenon, experimentally verified more than half a century ago, we hypothesize a local variability of the Planck constant (Kentosh et al., 2012a, 2012b; Flambaum et al., 2012) and impose, very intuitively, the conservation of energy (Cataldo, 2017a). Up until now, we have tacitly accepted the fact that mass is capable to warp space. Actually, if mass were to really warp space, we would be forced into admitting that, in a certain sense, the shape of the Universe can be modified with respect to something else, taken as reference. In the light of the foregoing remark, we may rather imagine that the value of space could be somehow modified by the presence of a gravitational source. Once accepted that a test particle, that we perceive as being punctual, is actually characterized by a radial extension, we could simply state that the more we approach the gravitational source, the more the value of the radial extension decreases. It has been previously claimed that the Universe we are allowed to perceive, when we are at rest, may be assimilated to a hypersphere. This assumption is not entirely correct: in fact, the space we perceive should be rather identified with a hyper-spherical shell, obviously characterized by a thickness (Cataldo, 2017c). In order to understand the previous assertion, suffice it to consider that we are undeniably used to identifying a paper sheet with a bidimensional surface. Nonetheless, we are well aware of the fact that a bi-dimensional surface represents nothing but a pure abstraction, and the above-mentioned sheet is evidently characterized by a thickness, whose value in no case should be considered as being null. We have to imagine the Planck constant as being linearly dependent on the dimensional thickness that, in turn, is linearly dependent on the radial coordinate (Cataldo, 2017a). In this way, by assigning a new meaning to the parameter usually identified with a Schwarzschild coordinate (our parameter does not represent a distance nor a radius of curvature) (Cataldo, 2017d), imposing the conservation of energy, we can obtain, without using General Relativity, the well-known expression for the Gravitational Redshift.

\section{Acknowledgements}

I would like to dedicate this paper to the memory of my father, Antonio Cataldo, who unexpectedly passed away on the $11^{\text {th }}$ of June 2016 .

\section{References}

Cataldo, C. (2016a). Faster than Light: again on the Lorentz Transformations. Applied Physics Research, 8(6), 1724. http://dx.doi.org/10.5539/apr.v8n6p17

Cataldo, C. (2016b). Effects of a Global Symmetry on the Observation of Astronomical Objects. Applied Physics Research, 8(5), 75-80. http://dx.doi.org/10.5539/apr.v8n5p75 
Cataldo, C. (2017a). A short discussion on the Gravitational Redshift in the light of an alleged local variability of the Planck Constant. Journal of Applied Mathematics and Physics, 5, 1001-1008. https://doi.org/10.4236/ jamp.2017.55087

Cataldo, C. (2017b). From General Relativity to A Simple-Harmonically Oscillating Universe, and Vice-Versa: a Review. Applied Physics. Research, 9(1), 86-92. http://dx.doi.org/10.5539/apr.v9n1p86

Cataldo, C. (2017c). From the Oscillating Universe to Relativistic Energy: a Review. Journal of High Energy Physics, Gravitation and Cosmology, 3, 68-77. http://dx.doi.org/10.4236/jhepgc.2017.31010

Cataldo, C. (2017d). Gravity and the Absoluteness of Time: a simple qualitative model. Applied Physics Research, 9(2), 42-52. http://dx.doi.org/10.5539/apr.v9n2pxx

Di Mauro, P., \& Notarrigo, S. (1995). Critica delle usuali derivazioni delle trasformazioni di Lorentz. Comunicazione al LXXXI Congresso Nazionale SIF, Perugia. Retrieved from http://www.lascuolaitalica.it/ pubbl12.htm

Di Mauro, P., \& Notarrigo, S. (1997). Sull'Invarianza delle Equazioni di Maxwell. Atti del XVI Congresso Nazionale di Storia della Fisica e dell'Astronomia, CNR Gruppo di lavoro per le Celebrazioni Voltiane, Como, 355-360. Retrieved from http://www.lascuolaitalica.it/pubbl5.htm

Flambaum, V. V., \& Berengut, J. C. (2012).Comment on "Global Positioning System Test of the Local Position Invariance of Planck's Constant". Physical Review Letters, 109, 068901. https://doi.org/10.1103/ PhysRevLett.109.068901

Ghosal, S. K., Nandi, K. K., \& Chakraborty, P. (1961). Passage from Einsteinian to Galilean Relativity and Clock Synchrony. Zeitschrift für Naturforschung, 46(a), 256-258. https://doi.org/10.1515/zna-1991-0307

Harrison, E. R. (1967). Classification of Uniform Cosmological Models. Monthly Notices of the Royal Astronomical Society, 137, 69-79. https://doi.org/10.1093/mnras/137.1.69

Kentosh, J., M., \& Mohageg, M. (2012a). Global Positioning System Test of the Local Position Invariance of Planck's Constant. Physical Review Letters, 108, 110801. https://doi.org/10.1103/PhysRevLett.108.110801

Kentosh, J., \& Mohageg, M. (2012b). Reply to Comment on "Global positioning system test of the local position invariance of Planck's constant”. Physical Review Letters, 109, 068902. https://doi.org/10.1103/PhysRevLett. 109.068902

Lorentz, H. A. (1904). Electromagnetic Phenomena in a System Moving with Any Velocity Smaller than That of Light. Proceedings of the Royal Netherlands Academy of Arts and Sciences, 6, 809-831. Retrieved from http://www.dwc.knaw.nl/DL/publications/PU00014148.pdf

Lorentz, H. A. (1909). The Theory of Electrons and Its Applications to the Phenomena of Light and Radiant Heat. Teubner. Retrieved from https://archive.org/details/electronstheory00lorerich

Maxwell, J. C. (1873). A Treatise on Electricity and Magnetism. Clarendon Press, Oxford. Retrieved from https://archive.org/details/electricandmagne01maxwrich

Schwarzschild, K. (1919). Über das Gravitationsfeld eines Massenpunktes nach der Einsteinschen Theorie. Sitzungsberichte der Deutschen Akademie der Wissenschaften zu Berlin, 189-196 (On the Gravitational Field of a Mass Point according to Einstein's Theory, translated by S. Antoci and A. Loinger, 1999). Retrieved from http://zelmanov.ptep-online.com/papers/zj-2008-03.pdf

Seshavatharam, U. V. S., \& Lakshminarayana, S. (2013a). Is Planck's Constant - A Cosmological Variable? Intern J. of Astronomy, 2(1), 11-15. Retrieved from http://article.sapub.org/10.5923.j.astronomy.20130201.02.html

Seshavatharam, U. V. S., Lakshminarayana, S., \& Sai, B. V. S. T. (2013b). Inadequacy of Modern Cosmology and Basics of Atomic Cosmology. viXra.org open e-Print archive. http://vixra.org/pdf/1303.0214v1.pdf

Voigt, W. (1887). Ueber das Doppler'sche Princip. Nachrichten von der Königlichen Gesellschaft der Wissenschaften und der Georg - Augusts - Universität zu Göttingen, Zeitschriftenband, 2(10), 41-51. Retrieved from http://www.digizeitschriften.de/dms/img/?PPN=PPN252457072_1887\&DMDID=dmdlog12

\section{Copyrights}

Copyright for this article is retained by the author(s), with first publication rights granted to the journal.

This is an open-access article distributed under the terms and conditions of the Creative Commons Attribution license (http://creativecommons.org/licenses/by/4.0/). 